\title{
Heterotopic ossification of the shoulder joint following spinal cord injury: an analysis of 21 cases after single-dose radiation therapy
}

\author{
M Citak ${ }^{1}$, D Grasmücke ${ }^{1}$, O Cruciger ${ }^{2}$, M Königshausen ${ }^{2}$, R Meindl $^{1}$, TA Schildhauer ${ }^{2}$ and M Aach ${ }^{1}$
}

\section{Study design: Retrospective chart review.}

Objectives: Heterotopic ossification ( $\mathrm{HO}$ ) affecting the hips is a common complaint of patients suffering traumatic spinal cord injury. However, the incidence of $\mathrm{HO}$ of the shoulder is considerably rare. In this context, we report on our results of 13 patients with a total of 21 cases of shoulder $\mathrm{HO}$ and single-dose radiation therapy.

Setting: Department of Spinal Cord Injury and Department of General and Trauma Surgery, BG-University Hospital Bergmannsheil Bochum, Ruhr-University Bochum, Germany.

Methods: The study group consists of 12 male and 1 female patient with a mean age of 55.5 years (range from 24 to 81 years; s.d. $=14.5$ ). Primary outcome measures were defined as the number of $\mathrm{HO}$ relapses and the number of side or adverse effects in relation to the radiation therapy.

Results: At the time of latest follow-up during hospitalization, the mean shoulder flexion was $92.1^{\circ}$ while mean abduction was $94.5^{\circ}$. The average external rotation was $26.4^{\circ}$. No HO recurrence occurred and none of the patients suffered any adverse effects related to radiation therapy.

Conclusions: In conclusion, single-dose radiation therapy in the treatment of shoulder $\mathrm{HO}$ due to spinal cord injury is an effective and reliable method, although the risk of secondary side effects related to the radiation therapy remain unknown.

Spinal Cord (2016) 54, 303-305; doi:10.1038/sc.2015.182; published online 27 October 2015

\section{INTRODUCTION}

Heterotopic ossification (HO) of the hips is a common complication after traumatic spinal cord injury (SCI). ${ }^{1-3}$ In contrast, neurogenic $\mathrm{HO}$ of the shoulder joint is a relatively rare clinical condition.,2,4 Genet and colleagues reported in a survey of 579 cases about their experiences of $\mathrm{HO}$ subsequent to central nervous system injuries. ${ }^{2}$ In this large cohort, the incidence of shoulder $\mathrm{HO}$ was 3.5\%. In a trial by Sautter-Bihl et al., ${ }^{4}$ the reported incidence of neurogenic shoulder $\mathrm{HO}$ was even lower with $2 \%$ in a cohort of 55 patients. Higher incidence of shoulder $\mathrm{HO}$ up to $4.9 \%$ subsequent to SCI have been reported by Citak et al. in a cohort of 132 patients. ${ }^{1}$

The minor occurrence of shoulder $\mathrm{HO}$ yields to a lack of evidence and/or literature analyzing the different therapy regimen. Recently, Pansard et al., reported on results of surgical intervention in a series of 19 shoulder HO cases in 16 patients after central nervous system injuries. In this particular group, patients developed troublesome shoulder $\mathrm{HO}$ after a mean time interval of 5.3 years post injury. ${ }^{5}$ Despite the reported good clinical outcomes reported by Pansard et al., surgical treatment may also be accompanied by intraoperative complications due to iatrogenic lesions of nerves and vessels. ${ }^{5}$ Therefore, early diagnosis of $\mathrm{HO}$ is crucial to avoid joint ankylosis and surgical intervention. In this context, we report on our screening and treatment regimen in SCI patients with shoulder $\mathrm{HO}$

\section{MATERIALS AND METHODS}

All SCI patients receiving treatment for shoulder $\mathrm{HO}$ with single-dose radiation therapy between January 2003 and December 2013 were included in this retrospective study. All patients being under age and/or without single-dose radiation therapy were excluded. Thirteen patients with a total of 21 manifestations of shoulder HO met the inclusion criteria for final analysis.

Diagnosis of $\mathrm{HO}$ was accomplished using our standardized hospital protocol. According to the protocol patients are screened biweekly by ultrasound examinations of the hip and shoulder joints by our experienced radiologists. In case of suspicious for $\mathrm{HO}$, the presumptive clinical diagnosis were confirmed with either computed tomography or magnetic resonance imaging. The extent of $\mathrm{HO}$ was classified according to the Brooker classification system. ${ }^{6}$ All patients with confirmed diagnosis received single-dose radiation therapy in cooperation with the radiation clinic.

\begin{abstract}
Data collection and outcome measures
The patient medical records provide the following information: age, gender, time interval of SCI and $\mathrm{HO}$ onset (in days), time interval of $\mathrm{HO}$ diagnosis and treatment with single-dose radiation therapy (in days), follow-up examination before discharge (in days) and site of $\mathrm{HO}$ localization. Primary outcome measures were the number of $\mathrm{HO}$ recurrences and number of side effects related to the radiation therapy. Relapse of $\mathrm{HO}$ was defined as the requirement of repeated radiation therapy due to $\mathrm{HO}$ progression. Only patients with clinical signs for $\mathrm{HO}$ are screened for $\mathrm{HO}$ recurrence using ultrasound examination or magnetic resonance imaging/computed tomography scan.
\end{abstract}

${ }^{1}$ Department of Spinal Cord Injury, BG-University Hospital Bergmannsheil, Ruhr-University Bochum, Bochum, Germany and ${ }^{2}$ Department of General and Trauma Surgery, BG-University Hospital Bergmannsheil, Ruhr- University Bochum, Bochum, Germany

Correspondence: Dr M Citak, Department of Spinal Cord Injury, BG-University Hospital Bergmannsheil, Ruhr-University Bochum, Bürkle-de-la-Camp Platz 1, 44789 Bochum, Germany.

E-mail: mcitak@gmx.de

Received 15 April 2015; revised 19 August 2015; accepted 10 September 2015; published online 27 October 2015 
Table 1 Demographic data and detailed information about concomitant injuries of patients with shoulder HO

\begin{tabular}{lcccll}
\hline Patient & Gender & Age & ASIA impairment scale & Neurological level of injury & Concomitant injuries \\
\hline 1 & M & 42 & A & C5 & None \\
2 & M & 49 & C & C7 & Thoracic trauma and bilateral humerus head fracture \\
3 & M & 52 & A & C6 & Thoracic trauma \\
4 & M & 63 & A & C6 & None \\
5 & M & 63 & A & C5 & Subarachnoid hemorrhage \\
6 & M & 75 & A & C6 & Thoracic trauma \\
7 & M & 59 & C & C5 & Tone \\
8 & M & 48 & A & Th4 & Thoracic trauma and craniocerebral trauma \\
9 & F & 62 & A & C5 & Thaniocerebral trauma \\
10 & M & 50 & A & C5 & Thoracic trauma and craniocerebral trauma \\
11 & M & 81 & C & C3 & Thoracic trauma and right clavicle fracture \\
12 & M & 25 & B &
\end{tabular}

Abbreviations: $\mathrm{F}$, female; $\mathrm{HO}$, heterotopic ossification; M, male.

Table 2 Detailed information of time interval of developing $\mathrm{HO}$ after $\mathrm{SCl}$ and time interval of detecting and treatment with single-dose radiation therapy of $\mathrm{HO}$. The table also reveals the $\mathrm{HO}$ localization side

\begin{tabular}{lcll}
\hline Patient & $\begin{array}{c}\text { Time interval between SCl and } \\
\text { development of shoulder HO }\end{array}$ & $\begin{array}{l}\text { Affected } \\
\text { side }\end{array}$ & $\begin{array}{c}\text { Time interval between } \\
\text { detecting and treatment }(d)\end{array}$ \\
\hline 1 & 30 & Bilateral & 5 \\
2 & 51 & Left & 4 \\
3 & 136 & Bilateral & 2 \\
4 & 142 & Bilateral & 12 \\
5 & 82 & Bilateral & 2 \\
6 & 69 & Right & 3 \\
7 & 131 & Bilateral & 2 \\
8 & 64 & Bilateral & 1 \\
9 & 24 & Right & 7 \\
10 & 50 & Bilateral & 4 \\
11 & 48 & Bilateral & 9 \\
12 & 43 & Right & 5 \\
13 & 62 & Right & 3 \\
\hline
\end{tabular}

Abbreviations: $\mathrm{HO}$, heterotopic ossification; $\mathrm{SCl}$, spinal cord injury.

Descriptive statistics are presented in the form of number of occurrences and percentage, or mean, s.d. and range. All data were processed using software (Graph Pad Prism version 5.0d, La Jolla, CA, USA).

\section{RESULTS}

The patient collective consists of 12 male and 1 female patient with a mean age of 55.5 years (range from 25 to 81 years; s.d.=14.5). The majority of the patients were tetraplegic $(n=11)$ and had an ASIA A lesion according to the American Spinal Injury Association (ASIA) Impairment Scale (AIS). ${ }^{7}$ Regarding concomitant injuries, only two patients suffered injuries around the shoulder joint (Table 1).

After a mean time interval of 71.7 days (range from 24 to 142 days; s.d. $=40.0)$ post SCI, shoulder HO occurred. In eight patients both shoulder joints were affected, while unilateral shoulder HO occurred in five patients. According to the Brooker classification, the two patients $(n=3)$ with concomitant injuries around the shoulder joints, had a Brooker IV. The remaining 15 cases had Brooker I and 3 patients were classified as Brooker II. All patients were treated with single-dose radiation therapy after a mean time interval of 4.5 days (range from 1 to 12 days; s.d.=3.2) after HO diagnosis (Table 2). In majority of cases (10 cases, $47.6 \%$ ) single-dose radiation therapy was performed with $7 \mathrm{~Gy}$ and $15 \mathrm{MV}$. In eight cases (38.1\%) $7 \mathrm{~Gy}$ and $6 \mathrm{MV}$ were administered. The remaining two patients were treated with $6 \mathrm{~Gy}$ and $10 \mathrm{MV}$.

At the final examination before discharge, with a mean follow-up time period of 88.8 days (range from 30 to 198 days; s.d. $=55.9$ ), average shoulder flexion was $92.1^{\circ}$ (range from 40 to $150^{\circ}$; s.d. $=30.0$ ) and mean abduction was $94.5^{\circ}$ (range from 45 to $140^{\circ}$; s.d. $=26.7$ ). Mean external rotation was $26.4^{\circ}$ (range from 10 to $40^{\circ}$; s.d. $=10.9$ ). No HO relapse occurred and none of the patients suffered any adverse effects related to the radiation therapy.

\section{DISCUSSION}

This is the first study reporting about the results of single-dose radiation therapy in the treatment of neurogenic shoulder $\mathrm{HO}$ in patients with acute SCI. As Pansard et al. reported, surgical resection, especially in the shoulder joint due to the anatomic relation to vessels and nerves, may lead to intra- and postoperative complications. ${ }^{5}$ Therefore, early diagnosis of $\mathrm{HO}$ is crucial to avoid joint stiffness and further surgical intervention.

In our study, we were able to achieve acceptable clinical results with single-dose radiation therapy. This may be accomplished by our standardized hospital protocol to diagnose $\mathrm{HO}$ in an early stage. Despite the standardized protocol, in three cases Brooker classification IV occurred. This most likely may be related to the fact that our spinal cord department is a tertiary referral center. Admission to our hospital subsequent to initial treatment is in some cases delayed.

As reported by Pansard et al., HO recurrence did not occur in our series. None of the patients suffered any side effects or complications related to the radiation therapy. However, it must be mentioned, that possible secondary side effects of radiation therapy may theoretically be possible. At this point, there exist no data reporting on the secondary side effects following radiation therapy in the treatment of $\mathrm{HO}$ in SCI patients, since the latency for tumor induction of radiation therapy is up to 27 years. ${ }^{89}$ Primary side effects after radiation therapy in the treatment of neurogenic $\mathrm{HO}$ have not been reported in the literature yet. ${ }^{4,10,11}$ 
Unfortunately, due to our sample of patients with severe impairments subsequent to SCI, it was not possible to use an established shoulder score such as constant or disabilities of the arm, shoulder and hand questionnaire score. Further limitations of the study are those related to the retrospective study design and the short follow-up time interval. A major limitation of the study is that only patients with clinical signs for $\mathrm{HO}$ were screened for $\mathrm{HO}$ recurrence. Therefore, patients who did not screen for HO recurrence could have theoretically $\mathrm{HO}$ and were in the non-HO recurrence group.

Despite those limitations, we could achieve acceptable clinical results using single-dose radiation therapy in the treatment of early $\mathrm{HO}$ of the shoulder joint. Using this method, we were able to treat all patients conservatively and could avoid the necessity of surgical resection with concomitant possible intra- or postoperative complications.

\section{DATA ARCHIVING}

There were no data to deposit.

\section{CONFLICT OF INTEREST}

The authors declare no conflict of interest.
1 Kedlaya D, Nazir C. Heterotopic ossification with normal serum alkaline phosphatase levels: a case series. Arch Phys Med Rehabil 2005; 86: E44.

2 Citak M, Kendoff D, O'Loughlin PF, Pearle AD. Heterotopic ossification post navigated high tibial osteotomy. Knee Surg Sports Traumatol Arthrosc 2009; 17: 352-355.

3 Wittenberg $\mathrm{RH}$, Peschke U, Botel U. Heterotopic ossification after spinal cord injury. Epidemiology and risk factors. J Bone Joint Surg Br 1992; 74: 215-218.

4 Sautter-Bihl ML, Hultenschmidt B, Liebermeister E, Nanassy A. Fractionated and single-dose radiotherapy for heterotopic bone formation in patients with spinal cord injury. A phase-I/II study. Strahlenther Onkol 2001; 177: 200-205.

5 Genet F, Kulina I, Vaquette C, Torossian F, Millard S, Pettit AR et al. Neurological heterotopic ossification following spinal cord injury is triggered by macrophagemediated inflammation in muscle. J Pathol 2015; 236: 229-240.

6 Brooker AF, Bowerman JW, Robinson RA, Riley LH Jr. Ectopic ossification following total hip replacement. Incidence and a method of classification. J Bone Joint Surg Am 1973; 55: 1629-1632.

7 Maynard FM Jr, Bracken MB, Creasey G, Ditunno JF Jr, Donovan WH, Ducker TB et al. International Standards for Neurological and Functional Classification of Spinal Cord Injury. American Spinal Injury Association. Spinal Cord 1997; 35: 266-274.

$8 \mathrm{Kim} \mathrm{JH}$, Chu FC, Woodard HQ, Melamed MR, Huvos A, Cantin J. Radiation- induced soft-tissue and bone sarcoma. Radiology 1978; 129: 501-508.

9 Ron E, Modan B, Boice JD Jr, Alfandary E, Stovall M, Chetrit A et al. Tumors of the brain and nervous system after radiotherapy in childhood. N Engl J Med 1988; 319: 1033-1039.

10 Citak M, Suero EM, Backhaus M, Meindl R, Schildhauer TA. Rhabdomyolysis after heterotopic ossification: an unusual complication in a spinal cord injured patient. Eur Spine J 2012; 21 (Suppl 4): S531-S534.

11 Sautter-Bihl ML, Liebermeister E, Heinze HG, Nanassy A, Stoltze D. The radiotherapy of heterotopic ossifications in paraplegics. The preliminary results. Strahlenther Onkol 1995; 171: 454-459. 